\title{
FATORES DE RISCO PARA O ENVOLVIMENTO DE JOVENS COM ORGANIZAÇÕES CRIMINOSAS
}

\author{
RISK FACTORS FOR INVOLVEMENT \\ OF YOUTH WITH CRIMINAL ORGANIZATIONS
}

Marcos Rolim ${ }^{1}$

Janaína Fassinato Pio de Almeida ${ }^{2}$

\begin{abstract}
Resumo
Sustentando a necessidade de se construir políticas de segurança pública com base em evidências, o artigo situa a realidade nacional marcada por altas taxas de homicídio e por práticas violentas que envolvem desproporcionalmente os jovens, como autores e vítimas preferenciais. Sumariza o paradigma dos fatores de risco da moderna Criminologia e expõe os resultados de duas revisões sistemáticas internacionais, realizadas recentemente, que efetuaram metanálise de estudos a respeito de fatores de risco para o envolvimento de jovens com organizações criminosas. Ambos os estudos encontraram correlações estatisticamente significativas para fatores de risco de natureza individual, familiar, escolar, de grupos de pares e comunitária, o que permite o delineamento de políticas públicas integradas com foco nesses fatores de forma a que o Poder Público se antecipe às dinâmicas produtoras da criminalidade e deixe de oferecer como resposta apenas as alternativas do Direito Penal.
\end{abstract}

\begin{abstract}
Supporting the need to build public security policies based on evidence, the article situates the national reality marked by high homicide rates and violent practices that disproportionately involve young people, as offenders and preferential victims. It summarizes the risk factor paradigm of modern Criminology and exposes the results of two recent international systematic reviews that conducted a meta-analysis of studies on risk factors for the involvement of young people with criminal organizations. Both studies found statistically significant correlations for risk factors of an individual, family, school, peer group and community nature, which allows the design of integrated public policies focusing on these factors so that the Public Power anticipates the dynamics producing crime and stop offering only the alternatives of Criminal Law as an answer.
\end{abstract}

\section{Introdução}

\footnotetext{
${ }^{1}$ Doutor e mestre em Sociologia, professor do mestrado em Direitos Humanos do Centro Universitário Ritter dos Reis - UniRitter

${ }^{2}$ Psicóloga, mestranda no curso de Direitos Humanos do Centro Universitário Ritter dos Reis - UniRitter
} 
O fenômeno do engajamento de jovens no crime, destacadamente no tráfico de drogas, alcançou enorme relevância nas sociedades contemporâneas e tem se reproduzido com especial virulência na América Latina. Já há muito, a Criminologia percebeu que a variável etária está fortemente correlacionada a práticas disruptivas. As evidências disponíveis mostram que a violência não se distribui equitativamente no conjunto social, mas que alguns grupos sociais estão muito mais sujeitos a ela, tanto na condição de vítimas quanto na de autores. Entre todos os grupos, adolescentes e jovens adultos do sexo masculino perfazem o conjunto mais suscetível (Hirschi \& Gottfredson, 1983).

A variável idade aparece com muita força nos estudos sobre envolvimento criminal, sendo que o intervalo compreendido entre a metade da adolescência e o início da idade adulta constitui o período crítico. A desproporcional concentração de atos violentos e criminais na juventude já havia sido percebida no século XIX pelo sociólogo belga Lambert Adolphe Jacques Quetelet (1796-1874). Em estudo sobre os registros criminais, ele identificou uma "idade de pico" para o envolvimento com atos delituosos e práticas violentas situado nos primeiros anos de vida adulta (Beirne, 1987). Em meados do século XX, os professores Sheldon e Eleanor Glueck, da faculdade de Direito de Harvard (EUA), conduziram um dos mais amplos e ambiciosos estudos longitudinais sobre fatores associados ao envolvimento criminal de jovens. Esse trabalho, “Desvendando a Violência Juvenil" (Unraveling Juvenile Delinquency), de 1950, confirmou que a idade era um fator chave na etiologia do crime. Os pesquisadores encontraram evidências tanto para a continuidade no crime, na vida adulta, quanto para o processo de desengajamento das opções delituosas. A grande maioria dos jovens que se envolve com o crime, sabemos hoje, abandona essa perspectiva nos primeiros anos da segunda década de vida em um processo de aparente "remissão espontânea" (Wolfang et $a l$, 1972). As evidências permitem concluir que, muito possivelmente, o fenômeno da desistência criminal (Rolim, 2018) seja condicionado por dois fenômenos básicos: a maturação dos indivíduos e a experiência de estabelecer os primeiros vínculos sociais como adultos, entre eles, o primeiro emprego, o casamento e a paternidade (Sampson \& Laub, 1995). Estudos de autorrelato (self-report studies), por seu turno, comprovaram que a esmagadora maioria das pessoas pratica, em algum momento de suas vidas frequentemente na adolescência e nos primeiros anos de vida adulta - atos delituosos, normalmente de menor gravidade. O que ocorre é que poucas dessas pessoas são flagradas 
quando do cometimento desses atos (Junger-Tas \& Marshall, 1999; Thornberry \& Krohn, 2000).

No caso brasileiro, desde o início da década de 80 pelo menos, temos experimentado uma espiral de violência com um envolvimento expressivo de jovens entre 14 e 25 anos. Apenas para ilustrar as dimensões do problema, bastaria lembrar que Waiselfisz (2013) encontrou que, entre 1980 e 2011, as mortes violentas de jovens (homicídios, suicídios e acidentes) cresceram 207,9\%. A elevação da taxa de homicídios entre os jovens, por seu turno, quando considerada isoladamente, foi de 326,1\% no período.

A resposta do Estado ao envolvimento dos jovens com o crime no Brasil têm sido, como se sabe, desde há muito, apenas aquela oferecida pelo Direito Penal. Para todos os efeitos, o que o Poder Público tem feito amplamente é encarcerar, uma resposta cujos efeitos iatrogênicos já foram amplamente identificados na literatura especializada (Nagin, 2010; Nagin, Cullen, \& Jonston, 2009).

O fenômeno contamina também as abordagens socioeducativas, quase sempre inviabilizadas pela demanda punitiva disseminada socialmente (Francischinni \& Campos, 2005). Nos últimos anos, essa mesma demanda alcançou maioria significativa na Câmara dos Deputados, o que redundou na aprovação da PEC 171/93 que propõe a alteração da redação do art. 228 da Constituição Federal para a imputabilidade penal do maior de dezesseis anos ${ }^{3}$. A PEC 171, a propósito, não ofereceu em sua justificativa uma única evidência científica em apoio à proposição de reduzir a idade penal. Seria mesmo difícil, porque as evidências disponíveis mostram que a medida ou é inócua ou pode agravar o cenário de violência, agenciando novas oportunidades para a precipitação dos vínculos criminais dos adolescentes. Em compensação, a justificativa da PEC trouxe três referências a personagens bíblicos: Salomão, Davi e Ezequiel. Quanto ao profeta Ezequiel, o autor da proposta assim se pronunciou:

A uma certa altura, no Velho Testamento, o profeta Ezequiel nos dá a perfeita dimensão do que seja a responsabilidade pessoal: Não se cogita nem sequer de idade: 'A alma que pecar, essa morrerá'.

A ideia de conter a criminalidade juvenil com a prisão desconsidera as evidências sobre os efeitos mais elementares do próprio processo de encarceramento. Estudos

\footnotetext{
${ }^{3}$ Matéria segue pendente de votação no Senado.
} 
clássicos como o de Blumenstein, Cohen \& Nagin (1978) mostraram, suficientemente, que políticas criminais centradas no encarceramento são, elas próprias, criminogênicas e agregam efeitos deletérios não apenas para os presos e seus familiares, mas para o conjunto da sociedade (Craig, 2012). O encarceramento em massa no Brasil tem sido dinamizado, entretanto, não como uma resposta específica à violência, mas como subproduto da política de "guerra às drogas", uma opção de política criminal que tem se demonstrado, em todos os lugares onde ela tem sido aplicada, não apenas inócua, mas contraproducente (Nosyk \& Wood, 2012).

Para se enfrentar os grandes desafios da redução da violência e da criminalidade é preciso superar a tradição, ainda hoje muito forte entre nós, de se lidar com políticas não amparadas por evidências científicas. O contencioso político-ideológico em curso no País tem agravado a incapacidade do Poder Público em delinear políticas de segurança com base em evidências, o que nos remete a um círculo vicioso onde fracassos colossais estimulam a demanda por mais doses das mesmas políticas que nos levaram ao fracasso, ao invés da exigência por novas abordagens.

Este artigo irá discutir os fatores de risco para o recrutamento de jovens por gangues e outras organizações criminosas, a partir das evidências encontradas por duas recentes Revisões Sistemáticas (Systematic Reviews)4. A definição de "gangue" nas Ciências Sociais não é pacífica, mas, na literatura especializada, tornou-se comum definir esse tipo de associação como grupos de jovens, entre 15 e 100 membros, na faixa dos 12 aos 24 anos, que compartilham elementos identitários como símbolos, tatuagens, cores ou territórios, com algum grau de organização e condensada performance criminal (Decker, Curry \& Pyrooz, 2013). No caso brasileiro, a definição caracteriza tipicamente os grupos de jovens das periferias associados às facções criminosas que disputam posições no mercado ilegal da venda de drogas.

Políticas públicas capazes de prevenir o crime e a violência precisam identificar os fatores de risco que operam sobre os grupos mais suscetíveis, de forma que seja possível que o Estado se antecipe ao engajamento criminal.

\footnotetext{
${ }^{4}$ Revisões sistemáticas são investigações de alta cientificidade que avaliam conjuntos de estudos sobre um mesmo tema, identificando quais são as evidências relevantes (Galvão \& Pereira, 2014). Um bom tutorial sobre revisões sistemáticas em português foi oferecido por Souza \& Ribeiro (2009).
} 


\section{Fatores de Risco: novo paradigma}

A Criminologia contemporânea tem produzido diferentes caminhos teóricos para a compreensão dos fenômenos criminais e violentos. A abordagem que trabalha com fatores de risco foi fortemente influenciada pelas evidências encontradas na área da saúde pública, notadamente na Epidemiologia. Assim, um novo paradigma criminológico foi desenvolvido (Farrington, 2000) a partir da identificação de fatores que aumentam as chances de envolvimento com o crime e a violência. Considera-se fator de risco qualquer característica, circunstância ou experiência que precede um resultado e que esteja correlacionada significativamente com esse resultado (Kazdin et al. 1997; Kraemer et al. 1997, 2001)

Fatores de risco exercem uma influência negativa na vida das pessoas, dos grupos sociais e das comunidades, favorecendo possibilidades disruptivas, promovendo a vitimização e o medo do crime na sociedade. São fatores preditivos para o engajamento criminal e para a experiência de vitimização quando lidamos com dados agregados. Esse detalhe é muito importante, porque correlações e a própria possibilidade de se identificar relações causais em fenômenos criminais só podem ser admitidas quando lidamos com dados estatisticamente significativos $(\mathrm{p}<0,05)^{5}$, o que exige a coleta de dados de grupos numericamente expressivos. No plano individual, a presença de fatores de risco apenas pode sugerir uma maior ou menor possibilidade de envolvimento criminal, o que é muito diferente de identificar determinações causais. Isso ocorre porque todas as pessoas também são expostas a fatores protetores (protective factors) que podem modular ou mesmo neutralizar os efeitos dos fatores de risco.

Fatores protetivos podem ser compreendidos como aquelas circunstâncias e/ou experiências que aumentam a resiliência diante das dificuldades e carecimentos e que promovem a segurança das comunidades. Por isso, alguém submetido na infância a fatores de risco não necessariamente deverá desenvolver comportamentos criminais ou violentos na vida adulta. $\mathrm{O}$ que os estudos têm demonstrado é que a grande maioria das pessoas que foram expostas a tais fatores não traduzirão essa influência em comportamentos disruptivos, possivelmente porque elas também foram expostas a fatores protetivos. Uma pequena parte, entretanto, fará esse percurso em direção à violência e ao

\footnotetext{
${ }^{5} \mathrm{O}$ valor da probabilidade é representado por "p". O nível de significância corresponde à evidência de que o fenômeno existe ou ao risco de rejeitar erradamente a hipótese nula.
} 
crime. Para se compreender melhor a importância dos fatores de risco, então, temos que lidar com os conceitos de prospecção e retrospecção. Quando projetamos os dados prospectivamente, os efeitos dos fatores de risco parecem pequenos, porque, do total de pessoas expostas, apenas uma pequena parte irá, de fato, se envolver com o crime e a violência. Entretanto, quando separamos aqueles que se tornaram violentos e que construíram carreiras criminais, vemos que, retrospectivamente, as correlações são muito fortes. Essa compreensão é decisiva para o delineamento de políticas eficientes de prevenção na área da segurança pública e, também, para evitar posições deterministas tendentes à estigmatização de pessoas e grupos sociais.

Os principais fatores de risco são de natureza individual, familiar, de grupo de pares, escolar e comunitária. Entre aqueles já identificados em estudos longitudinais, se poderia mencionar: baixa autoestima, impulsividade, dificuldades de concentração, estresse, abuso de álcool e/ou drogas, pobreza extrema, pais presos, vizinhança envolvida com o crime, ausência de modelos de conduta, baixa escolarização, experiência de vitimização na infância, negligência dos pais, testemunhar cenas de violência, desemprego, racismo, doenças mentais, famílias grandes, bullying na escola, evasão escolar, hiperatividade, comportamento antissocial na infância e na adolescência, etc.

No que se refere à intervenção possível por políticas públicas, sabe-se que programas focados em fatores de risco para a violência e o crime podem interrompê-los, reduzindo significativamente a chance de envolvimento de crianças e adolescentes na idade adulta (Hawkins, Catalano \& Miller 1992; Coie et al. 1993; Hawkins, Arthur \& Catalano 1995).

\section{Envolvimento de jovens com organizações criminosas: evidências de duas revisões sistemáticas}

O tema do recrutamento de jovens para o crime organizado ainda não originou uma tradição de pesquisas específicas no Brasil. Em muitos países, entretanto, já dispomos de muitos estudos longitudinais a respeito com base de dados colhidos randomicamente e com grupos de controle. Higginson et al (2018), em estudo de revisão sistemática pela Campbell, analisaram as evidências encontradas em oito estudos sobre 
fatores associados ao recrutamento de jovens por gangues em cinco países (Turquia, Trinidad e Tobago, El Salvador, China e Brasil) e na região do Caribe ${ }^{6}$.

A revisão teve dois objetivos básicos: a) sintetizar as evidências encontradas em artigos científicos (publicados ou não) sobre fatores de risco países de baixa e média renda para o recrutamento de jovens por grupos criminosos e b) avaliar a força relativa dos diferentes fatores de risco de natureza individual, familiar, escolar, de grupo de pares e comunitário. Os estudos selecionados para a revisão sistemática foram aqueles que atenderam aos requisitos estabelecidos previamente pelo Protocolo, todos com organizações criminosas juvenis em países de baixa ou média renda, com participantes entre 10 e 29 anos de idade, que identificaram preditores para a participação dos jovens nesses grupos, desde que os preditores tivessem sido individualizados e não considerados como um conglomerado. Todos os estudos incluídos na revisão deveriam, ainda, apresentar dados do grupo de jovens envolvidos e de grupo de controle formado por jovens com características similares sem envolvimento com o crime.

O exame dos oito estudos selecionados permitiu extrair 85 efeitos independentes de diferentes tamanhos com dados suficientes para criar um tamanho de efeito padronizado. Os autores da revisão calcularam o valor "d" de $\operatorname{Cohen}^{7}$ a partir de dados contínuos e a odds ratio ${ }^{8}$ dos dados dicotômicos. Todos os efeitos foram categorizados nos cinco tipos de fatores de risco e, depois, classificados em grupos conceitualmente semelhantes de fatores de risco ou protetivos. Os dados foram sintetizados com metanálises ${ }^{9}$ múltiplas de efeitos aleatórios com ponderação de variância inversa. Os resultados estão sumariados a seguir.

\section{a) Fatores de risco encontrados no plano individual}

\footnotetext{
${ }^{6}$ A pesquisa para a revisão sistemática foi realizada em inglês, francês, chinês, árabe, russo, espanhol e português. Foram realizadas buscas em 55 portais, incluindo bancos de dados acadêmicos, periódicos, além de sites governamentais, e não-governamentais que publicam estudos (grey literature), o que permitiu encontrar mais de 54 mil documentos para triagem.

7 Cálculo que permite analisar a diferença padronizada entre médias de dois grupos.

${ }^{8}$ Razão entre a chance de um evento ocorrer em um grupo e a chance de ocorrer em outro grupo.

9 Reproduzo a definição em português oferecida pela HTAnalyse; "Metanálise é o uso de métodos estatísticos para sumarizar resultados de estudos independentes. Combinando informações de todos os estudos relevantes, a metanálise pode estimar os efeitos de uma determinada intervenção de forma mais precisa do que cada estudo individualmente. Para a elaboração de uma revisão sistemática, é possível trabalhar com dados de diversas naturezas, contudo, para proceder com a abordagem meta-analítica, o resultado final de cada estudo deve ser expresso em uma medida padronizada, denominada tamanho do efeito" (Disponível em: https://www.htanalyze.com/metanalise/metanalise/ )
} 
1. Delinquência prévia

2. Uso do álcool, tabaco e maconha

3. Ser do sexo masculino,

4. Ter comportamento sexual de risco,

5. Estar em alguma atividade considerada de trabalho (legal ou ilegal)

6. Impulsividade/baixo autocontrole

7. Experiência de ter sido vitimado.

Os fatores protetivos identificados no plano individual foram todos de natureza psicológica e destacaram a empatia, a orientação para o futuro e a crença em uma ordem moral.

Jovens com baixa autoestima e que são impulsivos tem, aproximadamente, 50\% mais chances de se envolverem com grupos criminosos quando comparados com jovens que não possuem esses fatores de risco. Os jovens empáticos, que se orientam por um projeto de futuro e que acreditam na existência de uma ordem moral têm, aproximadamente, $40 \%$ menos chances de se envolver com gangues se comparados com os jovens que não possuem esses fatores psicológicos protetivos. Iniciação sexual precoce e ter mantido relações sexuais aparecem positivamente associadas com a participação dos jovens em gangues. Aqueles que tiveram essas experiências possuem três vezes mais chances de envolvimento com o crime, o que, no caso, possivelmente traduza comportamento impulsivo. O uso do álcool, do tabaco e da maconha também aparece correlacionado positivamente ao ingresso de jovens em gangues. O efeito geral combinado sugere que o consumo dessas drogas está correlacionado a mais que o triplo de chances de jovens pertencerem a gangues. O uso de drogas pesadas, por seu turno, pode aumentar em cinco vezes essa mesma probabilidade, mas essa correlação para drogas pesadas apareceu em apenas um dos estudos, de forma que não é forte o suficiente.

Não se verificou correlação significativa para o recrutamento relativos à idade, a pertencimento à etnia minoritária, a comportamento de proteção relacionado à conduta sexual ou ao uso de álcool ou uso moderado de drogas.

\section{b) Fatores de risco no grupo de pares}


Quanto ao tipo de fator de risco relacionado aos grupos de pares, a revisão encontrou evidência de maior probabilidade (quatro vezes mais chances) de envolvimento com gangues entre jovens que socializaram com pares envolvidos com comportamentos delinquentes.

\section{c) Fatores de risco no âmbito familiar}

1. Ambientes familiares negativos

2. Falta de supervisão dos filhos pelos responsáveis adultos

Ambientes familiares negativos foram considerados a partir de dados de situações de instabilidade familiar, da mudança de endereço no ano anterior e da experiência de fugir de casa. Ter uma vida familiar difícil pode quase dobrar os riscos de envolvimento criminal (92\% mais chances). Jovens que vivem com pai e mãe possuem a metade das chances de envolvimento com gangues quando comparados com aqueles que vivem com um só dos genitores. Considerando em conjunto os fatores protetivos, jovens que vivem em uma atmosfera familiar positiva, tem $25 \%$ menos chances de se envolver com gangues. A revisão não encontrou correlação significativa entre a entrada de jovens em grupos criminosos com famílias de baixos rendimentos, assim como com famílias de altos rendimentos. Uma correlação significativa foi encontrada, entretanto, com famílias de rendimento médio, mas de sentido preventivo ao envolvimento com o crime. Jovens que enfrentaram violência emocional ou física em suas famílias, que tiveram bens seus roubados ou danificados, que foram ameaçados ou feridos com uma arma ou que sofreram violência sexual têm o dobro de chances de se associarem a grupos criminosos.

Não foram encontradas correlações significativas entre a escolaridade dos pais e a entrada dos filhos em organizações criminosas, tampouco entre esse resultado e a atitude dos pais de legitimação de comportamentos antissociais.

\section{d) Fatores de risco escolares}

1. Baixo vínculo com a escola

2. Exposição à violência na escola

3. Dificuldades educacionais 
4. Oportunidades para o desenvolvimento pró-social.

Em geral, a exposição à violência na escola é um fator de risco significativo. Os jovens que passaram por esta experiência têm o triplo de chances de participação em gangues. Dificuldades na escola estão associadas ao envolvimento dos jovens com gangues, assim como baixo vínculo com a escola. Essa última experiência dobra os riscos de envolvimento com grupos criminosos. Por fim, o fato das "oportunidades de desenvolvimento pró-social" aparecerem correlacionadas à participação de jovens em gangues pode revelar correlação espúria, porque, muito possivelmente, escolas mais afetadas pela violência são as que oferecem mais comumente programas dessa natureza. Se esse for o caso, estaríamos, aqui, diante de uma falácia ecológica ${ }^{10}$.

Não foram encontradas correlações significativas para as variáveis escolarização, tipo de escola e desempenho acadêmico quanto aos jovens que foram recrutados por gangues.

\section{e) Fatores de risco comunitários}

A revisão encontrou altos níveis de correlação entre a experiência de ter sido exposto à violência na sua comunidade e a entrada em gangues. Assim como já se havia verificado para a violência familiar, jovens que enfrentaram violência emocional ou física nas suas comunidades, que tiveram bens roubados ou danificados, que foram ameaçados ou feridos com uma arma ou que sofreram violência sexual têm mais chances de se associarem a grupos criminosos (três vezes mais chances).

Outra revisão sistemática realizada por Frualdo (2019), incluiu 19 estudos sobre fatores de risco para o envolvimento de jovens com organizações criminosas, 10 desses estudos $(52 \%)$ foram desenvolvidos em países europeus, 7 (37\%) foram realizados na América do Norte, 2 dos estudos (11\%) foram realizados na América Latina. Uma definição mais aberta sobre grupos de crime organizado permitiu a inclusão de estudos sobre grupos mafiosos, grupos de traficantes, gangues e outros

\footnotetext{
${ }^{10}$ Interpretação equivocada de dados estatísticos quando se toma, por exemplo, características de um grupo não representativo como suficientes para deduções a respeito da população que constitui o universo pesquisado ou, no sentido contrário, quando se toma as características de um grupo e se presume que um indivíduo do grupo deva ter as mesmas características agregadas.
} 
Os estudos que foram elegíveis para a revisão encontraram 393 tamanhos diferentes de efeitos dos quais 155 (39\%) foram classificados como fatores psicológicos, $112(28 \%)$ como fatores sociodemográficos, 105 (27\%) relacionados à história criminal e apenas 21 deles (5\%) como fatores de status econômico. Seguindo os critérios metodológicos do estudo, foram elegíveis para a metanálise 56 tamanhos distintos dos efeitos.

Em síntese, a revisão encontrou que os membros das organizações criminosas eram, no geral, mais jovens que os indivíduos não-membros. Os que se vincularam às organizações criminosas estiveram menos expostos a fatores protetivos quando comparados com os grupos de controle, possuindo menor escolarização e não tendo maior envolvimento com religião. Foi constatada maior probabilidade desses jovens serem oriundos de famílias mais pobres e fragilizados e terem tido pouca supervisão por parte de um adulto quando crianças. Há mais chances desses jovens serem não-brancos e de terem amigos envolvidos em organizações criminosas. Em média, eles começaram mais precocemente no crime e são mais "produtivos" - no sentido de que cometem mais crimes - e versáteis.

Jovens recrutados pelo crime organizado têm mais probabilidade de terem envolvimento prévio com atos delituosos. Eles também têm mais chances de sofrimento psíquico e físico, enfrentando problemas como ansiedade, depressão, uso de drogas, ferimentos traumáticos e vitimização violenta. Ao mesmo tempo, esses jovens possuem mais chances de terem características psicológicas problemáticas e doenças que precederam seu envolvimento com o crime, como personalidade antissocial, baixo autocontrole, propensão a perseguir pessoas para aplicar o código violento das ruas, déficit nas funções cognitiva e executiva e psicopatia.

Assinale-se que os fatores de risco identificados nessa revisão não caracterizam, em regra, os grupos mafiosos. Jovens que apresentam baixo autocontrole, tendências violentas, déficit cognitivo e uso de drogas, por exemplo, são vistos como inconfiáveis por esse tipo de organização criminosa e tendem a não ser recrutados.

\section{Conclusão}

De todos os fatores de risco medidos pela revisão de Higginson et al (2018) as evidências mais fortes destacaram a experiência de delinquência prévia, o uso de álcool, tabaco e maconha, ser do sexo masculino e ter comportamento sexual de risco. A revisão 
de Frualdo (2019) encontrou fatores de risco que confirmam evidências acumuladas pela Criminologia. As duas revisões possuem pontos de contato importantes e evidenciam o quanto a etiologia do crime deve levar em consideração os diferentes caminhos que podem conduzir um jovem até a participação em uma organização criminosa.

O conhecimento sobre os diferentes fatores de risco correlacionados ao recrutamento de jovens por grupos criminosos e a identificação da natureza desses fatores nos planos individual, familiar, escolar, de grupo de pares e comunitário, por sua vez, permite que o Poder Público conceba políticas de segurança pública para muito além das capacidades e responsabilidades das instituições policiais.

Uma política de segurança cuja racionalidade seja a prevenção do crime e da violência deve se desdobrar em um plano de Estado capaz de articular políticas específicas que começam, na área da saúde pública, na forma como podem se realizar os exames de pré-natal e o acompanhamento da gravidez; se desdobram nos cuidados intensivos dos pais e no desafio de se criar em torno de cada bebê um ambiente de proteção, carinho e respeito; se prolongam nas atenções à primeira infância e na qualidade da educação infantil; seguem operando na escola e na possibilidade de se evitar o fracasso e a evasão; envolvem as estruturas comunitárias e as possibilidades de inserção dos jovens no mercado de trabalho; se relacionam com os valores preponderantes nos pares; com o racismo estrutural, com as diversas formas de exclusão e marginalização alavancadas por modelos econômicos injustos; com os preconceitos e estigmas disseminados socialmente; com as formas de abordagem que os policiais desenvolverão nas periferias, etc.

Em verdade, uma política de segurança eficiente haverá de seguir nos desafiando para as tarefas imprescindíveis de execução de medidas socioeducativas e para uma execução penal orientada pela ressocialização, de tal forma que, a partir de programas de prevenção terciária $^{11}$, sejamos capazes de resgatar os jovens que se associaram ao crime para as perspectivas cidadãs.

Esse é o caminho que se abre quando cogitamos de política de segurança delineadas com base em evidências científicas, ao invés de fundadas nos pantanosos terrenos das ideologias, das praxes institucionais, da demagogia ou da irresponsabilidade.

\footnotetext{
${ }^{11}$ Com a expressão se costuma caracteriza em Criminologia o tipo de programa ou política pública cujo foco são as pessoas que já se envolveram com o crime (Brantingham \& Faust,1976)
} 


\section{REFERÊNCIAS}

BEIRNE, Piers. Adolphe Quetelet and the Origins of Positivist Criminology. In: American Journal of Sociology 92(5): pp. 1140-1169, 1987.

BLUMENSTEIN, Alfred; COHEN, Jacqueline e NAGIN, Daniel (eds.) Deterrence and Incapacitation: Estimating the effects of Criminal Sanctions on Crime Rates. Washington, DC, National Academy of Sciences,1978..

BRANTINGHAM, Paul J. and FAUST, Frederic L. A Conceptual Model of Crime Prevention. Crime \& Delinquency, 22(3), 284-296, 1976.

COIE, John D., WATT, Norman F.; WEST, Stephen G; HAWKINS, J. David ; ASARNOW, Joan R.; MARKMAN, Howard J.; RAMEY, Sharon L.; SHURE, Myrna B. and LONG, Beverly. The Science of Prevention: A Conceptual Framework and Some Directions for a National Research Program. American Psychologist 48: 1013-22, 1993.

COUTO, Mônica Bonetti; OLIVEIRA, Simone Pereira de. GESTÃo DA JUSTIÇA E DO CONHECIMENTO: A CONTRIBUIÇÃO DA JURIMETRIA PARA A ADMINISTRAÇÃO DA JUSTIÇA. Revista Juridica, [S.1.], v. 2, n. 43, p. 771 - 801, fev. 2017. ISSN 2316-753X. Disponível em: <http://revista.unicuritiba.edu.br/index.php/RevJur/article/view/1869/1239>. Acesso em: 23 abr. 2020. doi:http://dx.doi.org/10.21902/revistajur.2316-753X.v2i43.1869.

DECKER, Scott H.; CURRY, G. David and PYROOZ, David C. Confronting Gangs: Crime and Community. Oxford University Press, 2013

FRACISCHINNI, Rosângela e CAMPOS, Herculano Ricardo. Adolescente em conflito com a lei e medidas socioeducativas: Limites e (im) possibilidades. Psico, 36(3), 267$273,2005$.

FRUALDO, Niccolò. A systematic review of factors leading to recruitment into organized crime. Tesi di laurea Facoltà di Scienze Politiche e Sociali Corso di Laurea in Politiche Pubbliche, Università Cattolica Del Sacro Cuore, 2019. Disponível em: https://www.researchgate.net/publication/334638859_A_systematic_review_of_factors _leading_to_recruitment_into_organized_crime

GALVÃO, Taís Freire e PEREIRA, Mauricio Gomes. Revisões sistemáticas da literatura: passos para sua elaboração. Epidemiol. Serv. Saúde, Brasília, 23(1):183-184, jan-mar 2014. Disponível em: http://www.scielo.br/pdf/ress/v23n1/2237-9622-ress-23-0100183.pdf

HANEY, Craig. Prison Effects in the Era of Mass Incarceration. The Prison Journal, 2012. Disponível em:

https://www.researchgate.net/publication/274998193_Prison_Effects_in_the_Era_of_M ass_Incarceration

HAWKINS, J. David, ARTHUR, Michael W. and CATALANO, Richard F. Preventing Substance Abuse. In Building a Safer Society: Strategic Approaches to Crime Prevention, 
edited by Michael Tonry and David P. Farrington, Vol. 19 of Crime and Justice: A Review of Research. Chicago: University of Chicago Press, 1995.

; CATALANO, Richard F. and MILLER, Janet Y. Risk and Protective Factors for Alcohol and Other Drug Problems in Adolescence and Early Adulthood: Implications for Substance Abuse Prevention. Psychological Bulletin 112: 64-105, 1992.

HIGGINSON, Angela; BENIER, Kathryn; SHENDEROVICH, Yulia ; BEDFORD, Laura ; MAZEROLLE, Lorraine and MURRAY, Joseph. Factors associated with youth gang membership in low and middle-income countries: a systematic review. A Campbell Systematic Review, 2018.

HIRSCHI, Travis; GOTTFREDSON, Michael. Age and explanation of Crime. American Journal of Sociology, 89: 552-584, 1983,

JUNGER-TAS, Josine \& MARSHALL, Ineke Haen. The Self-Report Methodology in Crime Research. Crime and Justice, Vol. 25, pp. 291-367, The University of Chicago Press.1999.

KAZDIN, Alan E., KRAEMER, Helena C.; KESSLER, Ronald C.; KUPFER, David J. and OFFORD, David R. Contributions of Risk-Factor Research to Developmental Psychopathology. Clinical Psychology Review 17: 375-406, 1997.

KRAEMER, Helena C.; KAZDIN, Alan E.; OFFORD, David R.; KESSLER, Ronald C.; JENSEN, Peter S. and KUPFER, David J. Coming to Terms with the Terms of Risk. Archives of General Psychiatry 54: 337-43, 1997.

KRAEMER, Helena C., STICE, Eric; KAZDIN, Alan; OFFORD, David and KUPFER, David. How Do Risk Factors Work Together? Mediators, Moderators, and Independent, Overlapping, and Proxy Risk Factors. American Journal of Psychiatry 158: 848-56, 2001.

NAGIN, D. S., CULLEN, F. T., and JONSTON, C. L. Imprisonment and reoffending. In M. Tonry, (Ed.), Crime and justice: An annual review of research (vol. 38, pp. 115200). Chicago: University of Chicago Press, 2009.

Imprisonment and crime control: Building evidence-based policy. In R. Rosenfeld, K. Quinet, \& C. Garcia (Eds.), Contemporary issues in criminological theory and research: The role of social institutions. Belmont, CA: Wadsworth, 2010.

NOSYK, B. and WOOD, E. Evidence-based drug policy: It starts with good evidence and ends with policy reform. International Journal of Drug Policy, 23 (6): 423-425, 2012. Disponível em:

https://www.ncbi.nlm.nih.gov/pmc/articles/PMC4570729/\#R21

ROLIM, Marcos. Desistência do crime. Sociedade e Estado, vol. 33, n 3, Brasília, set/dec 2018. https://doi.org/10.1590/s0102-6992-201833030008

SAMPSON, Robert J., LAUB, John H. Crime in the making: pathways and turning points through life. Cambridge, Massachusetts, Harvard University Press, 1995. 
SOUSA, Marcos R. de; RIBEIRO, Antonio Luiz P. Revisão sistemática e meta-análise de estudos de diagnóstico e prognóstico: um tutorial, Arq. Bras. Cardiol. vol.92 no.3 São Paulo Mar, 2009

THORNBERRY, Terence P. \& KROHN, Marvin D. The Self-Report Method for Measuring Delinquency and Crime in: Measurement and Analysis of Crime and Justice, Vol. 4 Criminal Justice. US Department of Justice, Office of Justice Programs 810 Seventh Street, N.W. Washington, DC, 2000.

WAISELFISZ, Julio Jacobo. Homicídios e Juventude no Brasil. Mapa da Violência 2013, Brasília, Secretaria-Geral da Presidência da República e Secretaria Nacional de Juventude, 2013.

WOlfGang, M.E., FIGLIO, R.M., SELlin, T. Delinquency in a Birth Cohort. Chicago: University of Chicago Press, 1972. 